\title{
Surgical Correction of Hirschsprung's Disease in Children Using the Soave-Boley Technique with Manual Colorectal Anastomosis
}

\author{
Oleh Kurtash ${ }^{*}$
}

\begin{abstract}
Introduction. Current trends in surgical treatment of Hirschsprung's disease are aimed at minimally invasive interventions. However, the experience of using Soave-Boley procedure in surgical treatment of Hirschsprung's disease in children of different ages is valuable in the arsenal of differentiated approach to the treatment of this pathology.

The objective of the research was to evaluate the results of surgical correction of Hirschsprung's disease in children using the Soave-Boley technique with manual colorectal anastomosis.

Materials and Methods. The analysis of surgical treatment of 1,187 children with different forms of Hirschsprung's disease aged from birth to 18 years over the period 1980-2020 was conducted in the National Children's Specialized Hospital "Okhmatdyt". Surgical correction of Hirschsprung's disease using the Soave-Boley technique was performed in 597 children. Before surgery, 156 patients underwent the first stage of treatment that consisted in the creation of a protective colostomy; in 441 cases, this intervention was performed without an intestinal stoma; the benefits of the Soave-Boley technique were evaluated.

Results. All the patients survived. In $15(2.51 \%)$ out of 597 children, in the early postoperative period, there were observed: retrocolic hematoma $(n=2)$, retrocolic abscess $(n=7)$, anastomotic leak $(n=2)$, adhesive intestinal obstruction $(n=3)$, intussusception $(n=1)$. Fourteen $(2.36 \%)$ patients developed surgical complications in the long-term period: residual aganglionosis $(n=9)$, anastomotic stenosis $(n=4)$ and coloptosis. Repeated Soave-Boley operation with manual colorectal anastomosis was successfully performed in $26(4.362 \%)$ patients after primary correction of Hirschsprung's disease using different methods. Periodic episodes of fecal smearing in the remote period in $45(7.53 \%)$ children were eliminated by conservative treatment. The success of this technique is confirmed by a much lower number of early $(2.51 \%)$ and late $(2.36 \%)$ postoperative surgical complications, as compared to those after using other methods of open surgical correction $-17.52 \%$ and $16.35 \%$, respectively.

Conclusions. Surgical correction of Hirschsprung's disease in children using the Soave-Boley technique with manual colorectal anastomosis allows better control over applying each suture, anatomical joining the edges of the bowel, and reducing trauma to the rectal mucosa, which ensures the high reliability of colorectal anastomosis; it is the most effective way of radical correcting Hirschsprung's disease in children in an open way in one-stage or two-stage interventions, and this technique is the operation of choice for secondary surgical correction of Hirschsprung's disease.
\end{abstract}

\section{Keywords}

Hirschsprung's Disease; Children; Surgical Treatment; Results

${ }^{1}$ Department of Pediatric Surgery, Ivano-Frankivsk National Medical University, Ukraine

*okurtash@ukr.net 


\section{Problem statement and analysis of the latest research}

Hirschsprung's disease (HD) is a complex malformation of the colon that may be treated only surgically. The essence of surgical correction is performing a resection of the aganglionic segment and a part of the altered intestine and imposing a colorectal anastomosis $[2,7]$.

Various methods of radical HD correction have been evolutionarily improved. The so-called open methods proposed by O. Swenson (1948), W. Duhamel (1956), F. Rehbein (1959), F. Soave (1963), H. Lynn (1956) and their numerous modifications appeared in chronological order [1, 6, 8, 14]. Each of these techniques has a specific goal in eliminating the causes and consequences of intestinal aganglionosis. As a result, the technique that is the least traumatic, the most technically acceptable, which eliminates all the pathogenetic factors and leads to the best functional results in the early and remote postoperative periods is acceptable for practical use. All these characteristics should certainly be accepted as a whole $[9,11,16]$.

Current trends in surgical treatment of HD are aimed at minimally invasive interventions. K.E. Georgeson proposed one-stage laparoscopy-assisted surgical treatment of HD. De La Torre described the first results of his own method, namely transanal endorectal pull-through (TEPT) [13, 14].

However, in some cases, open techniques are not rejected by pediatric surgery $[3,15]$. The Soave procedure modified by Boley is one of the most physiological and most technically acceptable technique among pediatric surgeons from different countries [5, 17]. Moreover, the main technical elements of this operation were included in the procedure of minimally invasive intervention in the correction of HD, namely TEPT. In addition, the Soave-Boley technique is a rational solution in conversion of minimally invasive interventions in patients with HD $[4,10,12]$.

Therefore, the experience of using the Soave-Boley procedure in surgical treatment of HD in children of different ages is valuable in the arsenal of differentiated approach to the treatment of this pathology.

The objective of the research was to evaluate the results of surgical correction of HD in children using the Soave-Boley technique with manual colorectal anastomosis.

\section{Materials and Methods}

The analysis of surgical treatment of 1,187 children with various forms of $\mathrm{HD}$ at the age from birth to 18 years over the period 1980-2020 was conducted (Table 1).

All the patients underwent both traditional and minimally invasive radical surgery. Fourteen children received the Swenson's pull-through operation; 29 children underwent the Duhamel procedure; 7 children were operated on according to the Rehbein's method; 59 children underwent the Soave procedure; 722 children underwent the Soave-Boley procedure. There was one more group which included 105 patients who underwent reconstructive plastic surgery in extensive forms of aganglionosis. Lynn's sphincteromyotomy was performed in 42 cases of ultra-short HD.

In addition, since October 2011, we have operated 209 children with HD using a minimally invasive technique. Among them, 144 patients were operated on according to TEPT approach and 65 children were operated on according to laparoscopy-assisted TEPT approach.

To diagnose and evaluate treatment outcomes during postoperative monitoring, the results of clinical examinations and special methods of investigation were used. They included radiological (irrigography, irrigoscopy, contrast radiography of the digestive tract) and endoscopic methods (sigmoidoscopy, colonoscopy, anorectal manometry), functional (anorectal manometry) and morphological studying (histological study, determination of acetylcholinesterase activity).

Having analyzed a 40-year experience in surgical treatment of HD according to different methods retrospectively, we considered necessary to study three periods, namely 1980 to 1992,1993 to 2010 and 2011 to 2020 each of which led us to approaches to improving the methods of surgical treatment of HD. It has become possible to modernize the methods of surgery, as well as to intensify the diagnostics for early detection of patients with HD (Table 2).

During the first period, we used so-called "open" surgeries, namely the Swenson, Duhamel, Soave procedures. During this period of our research, in the literature, there was a contentious debate over advantages and disadvantages of certain open methods. Therefore, we used different methods trying to reach a compromise in the differentiated approach to the selection of surgery.

In the second period, the main radical surgery we used was the Soave-Boley procedure modified by us with the implementation of manual or mechanical primary colorectal anastomosis (PCRA).

Since October 2011 (the third period), we have used minimally invasive techniques of HD surgical correction, namely TEPT and laparoscopy-assisted TEPT approach continuing to perform the Soave-Boley procedure in older children and those with a protective colostomy created during the first stage of surgery.

Reconstructive plastic surgery in extensive forms of aganglionosis and the Lynn procedure in uncomplicated ultra-short forms of $\mathrm{HD}$, as methods of correcting a separate category of patients, were performed during all study periods and did not depend on the main principles of division into these groups.

\section{Ethical Statement}

The research was carried out following the principles of the Declaration of Helsinki. The research protocol was approved by the Local Ethics Committee of Pediatric Surgery Clinic of the National Children's Specialized Hospital "Okhmatdyt". Informed parental consent was obtained for the research. 
Table 1. Distribution of patients according to age and type of aganglionosis.

\begin{tabular}{l|cccccc|cc}
\hline \multirow{2}{*}{ Type of aganglionosis } & \multicolumn{6}{|c|}{ Patients' age } & \multicolumn{2}{|}{ Total } \\
& $0-6$ months & $6-12$ months & $1-3$ years & $3-7$ years & $>7$ years & \multicolumn{2}{c}{$32.52 \%$} \\
Rectal & 39 & 61 & 74 & 95 & 117 & 386 & $32.38 \%$ \\
Rectosigmoid & 98 & 94 & 187 & 143 & 76 & 598 & $50.38 \%$ \\
Subtotal & 102 & 48 & 7 & 2 & 3 & 162 & $13.65 \%$ \\
Total colonic & 41 & - & - & - & - & 41 & $3.45 \%$ \\
\hline \multirow{2}{*}{ Total } & 280 & 203 & 268 & 240 & 196 & 1,187 & $100 \%$ \\
& $23.58 \%$ & $17.10 \%$ & $22.58 \%$ & $20.22 \%$ & $16.52 \%$ & & \\
\hline
\end{tabular}

Table 2. Distribution of patients according to variant of radical surgery during different periods of treatment modernization.

\begin{tabular}{l|ccc|c}
\hline \multirow{2}{*}{ Surgery } & \multicolumn{3}{|c|}{ Period (years) } & \multirow{2}{*}{ Total } \\
\cline { 2 - 4 } & 1 & 2 & 3 & \\
\hline Swenson procedure & $1980-1992$ & $1993-2010$ & $2011-2018$ & \\
Duhamel procedure & 14 & - & - & 14 \\
Rehbein procedure & 29 & - & - & 29 \\
Soave procedure & 7 & - & - & 7 \\
Lynn procedure & 59 & - & - & 59 \\
Manual Soave-Boley procedure & 14 & 21 & 7 & 42 \\
Soave-Boley procedure (by means of surgical stapler) & - & 344 & 253 & 597 \\
Reconstructive plastic surgeries in extensive forms of agan- & - & 125 & - & 125 \\
glionosis & 1 & 90 & 14 & 105 \\
TEPT & - & - & 144 & 144 \\
Laparoscopy-assisted TEPT & - & - & 65 & 65 \\
\hline Total & 124 & 580 & 483 & 1,187 \\
\hline
\end{tabular}

\section{Results and Discussion}

The main technical elements of operations to restore the patency of the intestinal tract in surgical treatment of children with HD are the mobilization of the colon and rectum and the formation of colorectal anastomosis. Modernizing these elements simplifies surgery, makes surgery less traumatic, facilitates the postoperative period, accelerates the rehabilitation period, and improves the patients' quality of life in the longterm period. Among the methods of open surgical management of HD, we prefer the Soave procedure modified by Boley as the most physiological and the most acceptable technique for the formation of manual colorectal anastomosis introduced in the Pediatric Surgery Clinic of the National Children's Specialized Hospital "Okhmatdyt". Gradually, the formation of mechanical (stapler) colorectal anastomosis was added to this technique. Since special equipment (circular stapler) is required to perform stapler entero-enteroanastomosis, manual colonic anastomosis was the most common in the clinic.

Manual Soave-Boley surgery was performed as follows: before the procedure, patients were placed in the lithotomy position; the abdominal cavity was opened with a wide midline incision up to the suprapubic region; a thorough examination of the abdominal organs was performed; the entire colon was examined; its aganglionic portion and suprastenotic part were visualized; the state of the enlarged intestine and the proximal margin of colon resection were determined.

During the examination of the colon, in most patients, the aganglionic area differed distinctly in diameter as compared to the area of the colon located above this site. The aganglionic area was pink, with longitudinal folds and sclerotic thickened wall. The state of suprastenotic colonic dilation depended on the length of aganglionosis, the degree of narrowing, the patient's age, the presence and severity of concomitant inflammatory process of the intestine.

If it was possible to achieve a good bowel movement before radical surgery, the area of suprastenotic dilation in such children was shorter, narrower, with more pronounced haustration and less hypertrophied intestinal walls in this area. Secondary changes in the supraganglionic zone were more pronounced in aganglionosis involving longer segments (above the level of the rectum), or after a long, ineffective conservative emptying of the intestine from fecal masses, or in case of protracted diagnosis of this pathology. The large intestinal segment, proximal to the narrowing site, was much wider, more haustric and sclerotic. This segment was pale pink; its mesentery was thinned, and stagnant purple blood vessels were significantly dilated. 
We mobilized the colon from the marking site to the transitional fold of the peritoneum, taking into account the architecture of the blood vessels and ureters.

Using the Soave technique, we separated a seromuscular cuff 1-3 cm above the transitional fold of the peritoneum (depending on the child's age).

In children, who first underwent protective colostomy creation, the seromuscular cuff was separated using the Soave technique. Then, we separated the colon stump in the form of a stoma from the anterior abdominal wall. The segment of the colon needed for constructing and anastomosis was mobilized.

The formation of the seromuscular cuff is the most difficult stage of surgery. Technical difficulties during this stage occurred due to pronounced sclerotic changes in the submucosal layer of the aganglionic zone that usually resulted from prolonged mechanical release of the intestine from fecal masses (the site of enema tip pressure), multiple dilations of the aganglionic area or prolonged influence of fecal masses before mechanical cleaning.

Significant sclerotic changes in the rectum sometimes led to damaging the mucous membrane during demucosation, which required careful treatment with antiseptics and suturing the defect. Demucosation was performed at the level of the dentate line of the rectum. Along the edges of the cuff, there were placed 2 clamps, between which the muscular layer of its posterior wall was dissected.

After that, the second surgical team of three people worked simultaneously to perform perineal surgery.

In children older than three years, the retrocolic space space was drained post-sphincterically through a separate skin incision using a PVC tube. The drainage was placed between the inner wall of the retrocolic space and the posterolateral surface of the rectum. Its introduction and placement were controlled visually from the side of the abdominal wound. The drainage was fixed with a separate ligature at the site of skin incision on the perineum. The purpose of draining the retrocolic space was to eliminate the exudate and possible hemorrhage from the site of the demucosed cuff. Patients older than three years are more likely to suffer from these fluid formations. The separation of the seromuscular cuff in children of this age group is the most traumatic. There is no need to drain the retrocolic space in children under three years of age.

After treating the lumen of the rectum with antiseptics, we inserted a fenestrated clamp into the anus, to which a loop of the mobilized colon was fixed. Using this clamp, we evaginated the mobilized colon through the seromuscular cuff towards the perineum. Thus, two cylinders were formed: the outer one - the mobilized mucous membrane of the rectum and the inner one - the evaginated colon. After complete evagination, we monitored the adequacy of rectal demucosation, a reliable sign of which is the presence of mobilized rectal crypts.

The evaginated rectal mucosa was further treated with an- tiseptics, dissected 2-4 $\mathrm{cm}$ below the mucocutaneous junction (depending on the patient's age), and fixed with four clamps.

After that, we continued to evaginate the colon to the marked level of proposed resection. At the same time, the surgeon of the team, who was working from the side of the abdominal cavity, carefully monitored the proper evagination of the colon.

We cut off all the layers of evagination step by step in a transverse direction $0.5-1.0 \mathrm{~cm}$ from the dentate line and applied sutures (PDS, vicryl 3/0-4/0) circularly between all the layers of the colon and the rectal mucosa, thereby forming colorectal anastomosis.

After applying anastomosis, we inserted a tube into the lumen of the evaginated colon above the line of the conjunction to remove gases and feces in the postoperative period.

The rectal tube was fixed with a ligature to the skin of the buttocks on the opposite side from the place of fixation of the retrocolic drainage.

All the patients survived. In $15(2.51 \%)$ out of 597 children, in the early postoperative period, there were observed surgical complications: retrocolic hematoma $(n=2)$, retrocolic abscess $(n=7)$, anastomotic leak $(n=2)$, adhesive intestinal obstruction $(n=3)$, intussusception $(n=1)$. Retrocolic hematoma was conservatively corrected in both patients. In case of retrocolic abscess and anastomotic leak, it was necessary to impose a right-sided protective colostomy and to proceed conservative treatment of the abscess and leakage. We closed colostomy after 2-4 months in case of complete healing of all complications and the absence of severe anastomosis stenosis. Relaparotomy and viscerolysis were performed in case of adhesive intestinal obstruction. Intussusception was corrected by disinvagination in repeat laparotomy.

Fourteen $(2.36 \%)$ patients developed surgical complications in the long-term period: residual aganglionosis $(\mathrm{n}=$ $9)$, anastomotic stenosis $(n=4)$ and coloptosis. All children with residual aganglionosis underwent sphincteromyotomy. Colorectal anastomotic stenosis was corrected by bougienage in three children. Repeated Soave-Boley surgery with colorectal anastomosis was performed manually in another patient to eliminate anastomotic stenosis. In coloptosis, which was the cause of recurrent constipation and abdominal pain, there was performed relaparotomy with resection of the transverse colon.

Repeated Soave-Boley operation with manual colorectal anastomosis was successfully performed in $26(4.362 \%)$ patients after primary correction of HD in other clinics using different methods. The reasons for re-correction of HD were as follows: residual aganglionosis $(\mathrm{n}=1)$ after the Rehbein procedure; residual aganglionosis $(n=2)$ and the presence of a "sail" between the stumps of the rectum and colon at the site of colorectal anastomosis $(n=2)$ after the Duhamel procedure; residual aganglionosis $(n=2)$ after the classic Soave procedure; residual aganglionosis $(\mathrm{n}=10)$ and stenosis of colorectal anastomosis $(n=6)$ after the Soave-Boley operation with manual colorectal anastomosis; residual aganglionosis $(\mathrm{n}=2)$ after 
the Lynn procedure (sphincteromyotomy); anastomotic stenosis $(n=2)$ after laparoscopy-assisted TEPT. The technical capabilities and the results of the early and long-term periods allowed us to assert that the Soave-Boley technique with manual colorectal anastomosis is the operation of choice for secondary surgical correction of HD by any other means.

Periodic episodes of fecal smearing in the remote period in $45(7.53 \%)$ children were eliminated by conservative treatment, which was carried out in specialized centers. Moreover, the psychological state and the persistence of parents and patients played a significant role in the adaptation period. The goal of HD treatment consisted in both medical and social rehabilitation of children, providing their early adaptation in society.

In general, the experience in treating HD with other methods of open surgical correction (the Swenson's pull-through operation $(n=14)$, the Duhamel procedure $(n=29)$, the Rehbein method $(n=7)$ and the Soave procedure $(n=59)$ ) helps conclude that the Soave-Boley procedure with manual colorectal anastomosis has significant technical advantages. The Soave-Boley technique allows better control over applying each suture and anatomical joining the edges of the rectum and colon. This reduces trauma to the rectal mucosa, contributes to the high reliability of colorectal anastomosis formation. The success of this technique is confirmed by a much lower number of early $(2.51 \%)$ and late $(2.36 \%)$ postoperative surgical complications, as compared to those after using other methods of open surgical correction $-17.52 \%$ and $16.35 \%$, respectively.

\section{Conclusions}

1. The method of surgical correction of HD in children by the Soave-Boley technique with manual colorectal anastomosis allows better control over applying each suture, anatomical joining the edges of the bowel, and reducing trauma to the rectal mucosa, which ensures the high reliability of colorectal anastomosis.

2. According to the technical capabilities and the results of the early and long-term periods, the Soave-Boley technique with manual colorectal anastomosis is the operation of choice for secondary surgical correction of HD by any other methods.

\section{Conflict of Interest}

The authors declare that no conflicts exist.

\section{Financial Disclosure}

The authors declared no financial support.

\section{References}

[1] Kryvchenia DYu, Prytula VP, Silchenko MI, Danshyn TI, Sitkovska SM, Matiiash OYa. Rezultaty likuvannia ditey z khvoroboiu Hirshprunha. Prohnozy ta shliakhy pokrashchennia. Paediatric Surgery. 2008;4:51-54.

[2] Prytula VP, Silchenko MI, Hussaini SF, Kuzyk AS, Kurtash OO, Iskov AP. Transanal endorectal pull-through and resection of colon, as modern approach to the treatment of Hirschprung's disease in chidren. Archive of Clinical Medicine. 2014;20(2):93-94.

[3] Peng C-H, Chen Y-J, Pang W-B, Zhang T-C, Wang ZM, Wu D-Y, et al. STROBE-anastomotic leakage after pull-through procedure for Hirschsprung disease. Medicine [Internet]. 2018 Nov;97(46):e13140. Available from: https://doi.org/10.1097/MD.0000000000013140

[4] Ekenze SO, Ngaikedi C, Obasi AA. Problems and Outcome of Hirschsprung's Disease Presenting after 1 Year of Age in a Developing Country. World Journal of Surgery [Internet]. 2010 Oct 26;35(1):22-26. Available from: https://doi.org/10.1007/s00268-010-0828-2

[5] Fernández Ibieta M, Sánchez Morote JM, Martínez Castaño I, Cabrejos Perotti K, Reyes Ríos P, Rojas Ticona J, et al. [Quality of life and long term results in Hirschsprung's disease]. Cir Pediatr [Internet]. 2014 Jul;27(3):117-24. Available from: http://www.ncbi.nlm.nih.gov/pubmed/25845100

[6] Friedmacher F, Ure B, Lacher M, Gosemann J-H. Open Versus Transanal Pull-Through for Hirschsprung Disease: A Systematic Review of Long-Term Outcome. European Journal of Pediatric Surgery [Internet]. 2013 Apr 9;23(02):094-102. Available from: https://doi.org/10.1055/s-0033-1343085

[7] Khademi G, Khazdouz M, Sezavar M, Imani B, Akhavan $\mathrm{H}$, Babapour A. Clinical outcome and bowel function after surgical treatment in Hirschsprung's disease. African Journal of Paediatric Surgery [Internet]. 2015;12(2):143. Available from: https://doi.org/10.4103/01896725.160403

[8] Langer JC. Hirschsprung disease. Current Opinion in Pediatrics [Internet]. 2013 Jun;25(3):368-374. Available from: https://doi.org/10.1097/MOP.0b013e328360c2a0

[9] Lee J-T, Liu C, Tsai H-L, Chin T, Wei C-F. Successful Redo Pull-through for Hirschsprung's Disease in a Haddad Syndrome Patient. Journal of the Chinese Medical Association [Internet]. $2010 \mathrm{Aug} ; 73(8): 438-440$. Available from: https://doi.org/10.1016/S1726-4901(10)70094-0

[10] Levitt MA, Dickie B, Peña A. The Hirschsprungs patient who is soiling after what was considered a "successful" pull-through. Seminars in Pediatric Surgery [Internet]. $2012 \mathrm{Nov} ; 21(4): 344-353$. Available from: https://doi.org/10.1053/j.sempedsurg.2012.07.009 
[11] Yang L, Tang S, Cao G, Yang Y, Li S, Li S, et al. Transanal endorectal pull-through for Hirschsprung's disease using long cuff dissection and short Vshaped partially resected cuff anastomosis: early and late outcomes. Pediatric Surgery International [Internet]. 2012 Mar 20;28(5):515-521. Available from: https://doi.org/10.1007/s00383-012-3071-0

[12] Neuvonen MI, Kyrklund K, Rintala RJ, Pakarinen MP. Bowel Function and Quality of Life After Transanal Endorectal Pull-through for Hirschsprung Disease. Annals of Surgery [Internet]. 2017 Mar;265(3):622-629. Available from: https://doi.org/10.1097/SLA.0000000000001695

[13] Prytula VP, Levytskyi AF, Silchenko MI, Hussaini SF, Godik OS, Kurtash OO, et al. Laparoscopicassisted transanal endorectal pull-through of colon for treatment of Hirschsprung's disease in children. Standardy Medyczne-Problemy Chirurgii Dzieciecej. 2016;6(1):109.

[14] Sheng Q, Lv Z, Xiao X. Re-operation for Hirschsprung's disease: experience in 24 patients from China. Pediatric Surgery International [Internet]. 2012 Feb 23;28(5):501-506. Available from: https://doi.org/10.1007/s00383-012-3062-1
[15] Tabbers MM, DiLorenzo C, Berger MY, Faure C, Langendam MW, Nurko S, et al. Evaluation and Treatment of Functional Constipation in Infants and Children. Journal of Pediatric Gastroenterology and Nutrition [Internet]. $2014 \mathrm{Feb}$;58(2):265-281. Available from: https://doi.org/10.1097/MPG.0000000000000266

[16] Mahler T, Dassonville M, Truong D, Robert A, Goyens P, Steyaert H, et al. Long-Term Outcomes and Quality of Life in Patients after Soave Pull-Through Operation for Hirschsprung's Disease: An Observational Retrospective Study. European Journal of Pediatric Surgery [Internet]. 2017 Jul 24;28(05):445-454. Available from: https://doi.org/10.1055/s-0037-1604115

[17] Wilkinson DJ, Edgar DH, Kenny SE. Future therapies for Hirschsprung's disease. Seminars in Pediatric Surgery [Internet]. $2012 \mathrm{Nov} ; 21(4): 364-370$. Available from: https://doi.org/10.1053/j.sempedsurg.2012.07.010

Received: 2020-06-04

Revised: 2020-08-31

Accepted: 2020-11-01 